\title{
Long-Term Impact of Caregiving and Metabolic Syndrome with Perceived Decline in Cognitive Function 8 Years Later: A Pilot Study Suggesting Important Avenues for Future Research
}

\author{
Beverly H. Brummett ${ }^{1}$, Shirley B. Austin ${ }^{1}$, Kathleen A. Welsh-Bohmer ${ }^{2}$, Redford B. Williams ${ }^{1}$, \\ Ilene C. Siegler ${ }^{1}$ \\ ${ }^{1}$ Department of Psychiatry and Behavioral Medicine, Duke Medical Center, Durham, USA \\ ${ }^{2}$ Joseph and Kathleen Bryan Alzheimer's Disease Research Center, Durham, USA \\ Email: brummett@duke.edu
}

Received November 21, 2012; revised December 31, 2012; accepted January 9, 2013

\begin{abstract}
The chronic stress of caregiving has been associated with increased risk for cognitive decline and dementia. One theoretical model suggests that a group of risk factors known as the metabolic syndrome MET_SYN (e.g. hypertension, poor glucose regulation, central obesity, and high triglyceride levels) that have demonstrated associations with both stress and cognitive decline, may mediate the association between caregiver stress and cognitive decline. It is also possible that caregiving may moderate the association between MET_SYN and cognitive decline. The present study examined these two potential models. The study sample consisted of 53 caregivers for a relative with dementia and 24 participants who did not have caregiving responsibilities at baseline. We examined associations among caregiving history (yes/no), self-reported decline in cognitive function (the AD8) at follow-up, and a MET_SYN factor comprised of increased systolic blood pressure (SBP), glycosylated hemoglobin concentration (HbA1c), waist circumference, and triglyceride levels at baseline when caregiving was assessed. MET_SYN was associated with AD8 $(p=0.010)$. Caregiving history was not directly associated with AD8 ratings, however, caregiving did moderate the association between MET_SYN and AD8 $(p=0.043)$ assessed 8 years later. In caregivers MET_SYN scores reflecting higher risk were associated with scores on the AD8 indicting decline, whereas, in controls MET_SYN was unrelated to AD8 assessment. Thus, it can be concluded that caregiver stress may increase the association between metabolic risk factors and decline in cognitive functioning up to 8 years later.
\end{abstract}

Keywords: Caregiving; Metabolic Syndrome; Cognitive Decline

\section{Introduction}

The chronic stress associated with caregiving for a loved one with dementia has been shown to increase rates of cognitive decline e.g. [1-3], and risk of incident risk in the caregiver [4]. Caregiving stress also has an adverse effect on cardiovascular risk factors that characterize the metabolic syndrome (MET_SYN) (e.g. poor glucose blood pressure regulation, central obesity, and hypertension) $[5,6]$. Because the MET_SYN has been linked to increased rates of cognitive decline [7-9], it has recently been hypothesized that the impact of caregiving on cognitive decline is mediated via its association with the MET_SYN [10].

Within the large body of literature that has focused on the role that chronic stress plays with regard to illness, it is known that certain stressors may trigger disease pro- cesses in some individuals, and those same stressors may not create distress in others [11]. For example, emotional stress to an anger induction has been shown to heighten cortisol response in genetically vulnerable individuals, while cortisol response remained unaffected by this stressor in other individuals [12]. In related work demonstrating how stress may moderate the relations among psychosocial factors and disease, caregiving was associated with poor glucose regulation, with effects dependent on neighborhood characteristics [13]. Specifically, neighborhood stress was related to poorer glucose regulation, but only among caregivers and not among controls. Such findings highlight the need to consider the possibility that stressors may serve as moderators, and thus it is critical to consider both mediation and moderation models when examining the relations among stress, risk factors, and disease processes. 
Based on the foregoing, the current study was undertaken to test two hypotheses: A. The impact of caregiving on an indicator of cognitive decline will be mediated by increased expression of the MET_SYN (i.e., higher resting systolic blood pressure (SBP), glycosylated hemoglobin concentration (HbA1c), waist circumference, and triglyceride levels); and B. The impact of MET_SYN on cognitive decline will be enhanced (moderated) by caregiving stress.

Psychosocial, behavioral, and metabolic risk factors were assessed in a sample of 175 caregivers and 169 controls in a study completed between 2001 and 2004. A follow-up study conducted between 2010 and 2011 reenrolled 80 of these participants (53 caregivers and 27 age/race matched non-caregiver controls). The follow-up study was designed to assess the relations among caregiving, metabolic risk factors, and mild decline in cognitive functions. These 80 participants completed a selfreport measure of cognitive functioning, the AD8. This measure has been shown to differentiate non-demented from demented individuals, and to distinguish mildly impaired individuals from those who are more demented [14]. In the present study MET_SYN was assessed at baseline and approximately 8 years later perceived cognitive decline (AD8) was assessed. This length of followup ensured an extended period of caregiving prior to the assessment of cognition. To examine our proposed mediation and moderation models, we conducted analyses that examined the direct relations among caregiving (yes/ no), a metabolic risk factor MET_SYN (comprised of resting SBP, HbA1c, waist circumference, and triglyceride levels), and AD8 scores. In addition, we examined caregiving as a potential moderator of relations among MET_SYN and AD8.

\section{Methodology}

\subsection{Sampling Procedures}

Baseline Study: Participants were recruited to be part of a study designed to examine the underlying biological and behavioral mechanisms whereby stressful social and physical environments lead to health disparities. Caregivers were recruited using flyers, ads in the local media, and community outreach efforts. Non-caregiver controls were recruited by asking caregivers to nominate two to five friends who live in their neighborhood and are similar with respect to demographic factors (i.e., gender, age, and race). Baseline data were collected in two venues-a questionnaire battery was given to participants during a home visit by a nurse and returned upon their visit to the General Clinical Research Center (GCRC) at Duke University Medical Center. During the clinic visit, participants received a general physical examination at which time blood pressure was taken, waist circumference was assessed, and blood was drawn for assessment of glucose and lipid metabolism. The baseline sample consisted of 344 participants, 175 caregivers and 169 non-caregiver controls, matched on age, race, and socioeconomic status.

Follow-up Study: The follow-up study, funded by the Joseph and Kathleen Bryan Alzheimer's Disease Research Center at Duke University Medical Center, was designed to collect data on 80 participants from the baseline study who had given permission to be contacted at a later date for possible follow-up studies. The follow-up study gathered information on cognitive function, current caregiving responsibilities, and self-report of current health status. The present sample consisted of 54 caregivers and 23 non-caregivers (3 control participants were excluded due to the fact that they were now serving as a primary caregiver). In the approximate 8 years between baseline and follow-up, a majority of caregivers experienced the death of their care recipient, however, all of the caregiver participants had experienced the stress of being the primary caregiver for a relative with dementia for a number of years and 70\% had been a non-caregiver for a period of less than 5 years. Both the baseline and the follow-up studies were approved by the Duke University Medical Center Institutional Review Board.

\subsection{Measures}

\subsubsection{Demographic Measures}

Age was represented in years. Race was self-identified and all individuals were either African American or Caucasian.

\subsubsection{Clinical Measures}

Glycosylated hemoglobin concentration (HbA1c\%) was assayed in all participants. HbA1c is the standard surrogate marker for measurement of average plasma glucose over the past three months [15]. HbA1c has been related to stress in patients with diabetes [16-18], to cardiovascular mortality and morbidity [19], and to the outcome of stroke [20]. HbA1c was determined at the Franklin Site Laboratory (Duke University Medical Center) by ion based high performance liquid chromatography, using a Tosoh (version G7) analyzer.

Triglycerides. Serum triglyceride levels (mg/dL) were assayed by the CDC-approved laboratory facility at Lab Corp in Burlington, NC.

Waist Circumference and Blood Pressure. Waist circumference and resting blood pressure and were measured during the physical examination administered by the research study nurse. Waist circumference $(\mathrm{cm})$ was measured by a nurse to the nearest $0.5 \mathrm{~cm}$ with the subjects standing, using a nonexpendable linen tape measure, according to the American Heart Association recommendations. 


\subsubsection{Cognitive Function}

The AD8 dementia screening interview was used to assess decline in cognitive function [14,21]. The AD8 can be used as an informant interview, or as a self-rating tool to detect dementia [14,21]. Briefly, individuals are asked whether or not there has been a change in the last several years caused by cognitive (thinking and memory) problems. Individuals respond to 8 probes in the following categories: 1) problems with judgment; 2) less interest in hobbies/activities; 3 ) repeating the same things over and over; 4) trouble learning how to use a tool, appliance, or gadget; 5) forgetting correct month or year; 6) trouble handling complicated financial affairs; 7) trouble remembering appointments; 8) daily problems with thinking and/or memory. Items rated as "yes, a change", or "no, no change", and the sum of "yes" items reflects the total score. Thus, higher score is indicative of a decline in cognitive function over the past several years, and a score of 0 would reflect no change over the interval.

\subsection{Statistical Analyses}

Initially, a principal components factor analysis was conducted to derive a factor score that would represent MET_SYN in later analyses. Table 1 presents the factor loadings for each of the measures in the factor (SBP, HbA1c, waist circumference, and triglyceride levels). A higher MET_SYN score indicates higher levels for each risk factor.

Regression analyses were used to examine caregiving and MET_SYN as predictors of AD8 ratings, as well as to test MET_SYN as a potential mediator of observed relations between caregiving and AD8. To examine moderation by caregiving, a regression model was conducted that contained a term representing group (caregiver status yes/no), the factor score representing MET_SYN, and the interaction term group $x$ MET_SYN as predictors of AD8 scores. Analyses included age, race, and gender as adjustment covariates.

SAS V8 statistical software (SAS Institute: Cary, NC) was used to conduct all analyses. Data were examined for potential outliers and values that were $3 \mathrm{SD}$ or more above the mean for any given measure were recoded to a

Table 1. Metabolic syndrome (MET_SYN) loadings from principal components factor analysis.

\begin{tabular}{cc}
\hline Metabolic risk factor & Loading \\
\hline Resting systolic blood pressure & 0.80 \\
Glycosylated hemoglobin concentration (HbA1c\%) & 0.75 \\
Waist circumference (cm) & 0.50 \\
Serum triglyceride (mg/dL) & 0.45 \\
\hline
\end{tabular}

value reflecting the $99 \%$ for that measure. The significance level for all analyses was $p<0.05$.

\section{Results}

The present sample of 77 participants was $79.2 \%$ female; $53.3 \%$ Caucasian, and had a mean age of 62.4 (SD 10.5). The means (SD) for the measures comprising the MET_SYN factor were: SBP = 136.6 (22.0); HbA1c = 5.8 (1.1); waist circumference $\mathrm{cm} 95.5$ (17.4); and triglycerides 117.3 (68.5). AD8 ratings had a mean of 1.2 (SD 1.5, range 0 - 6).

MET_SYM was significantly related to AD8 scores ( $p=0.010)$, whereas caregiving was not significantly related to AD8 scores $(p=0.618)$. Therefore, mediation analyses were not conducted due to the nonsignificant relation between caregiving and AD8 scores.

The caregiving group $x$ MET_SYN interaction was a significant predictor of AD8 $(p=0.043)$. Within group follow-up correlation analyses were used to examine the direction and the strength of observed relations between the AD8 and the MET_SYN factor, in caregiver and control groups. In caregivers higher MET_SYN scores were strongly associated ( $r=0.46, p=0.001)$ with scores on the AD8 indicting decline, whereas, in controls MET_SYN was not significantly unrelated $(r=0.11, p=$ $0.66)$ to AD8 assessment. Figure 1 presents the relation between MET_SYN and AD8 by group.

\section{Discussion}

Existing theories suggest that caregiving may be associated with decline in cognitive function and dementia risk, with potential mediation by metabolic risk factors, or alternatively, that caregiving may moderate associations between metabolic factors and cognitive decline. While not supporting the mediation hypothesis the present findings do support a moderation hypothesis, indicating that in caregivers, but not controls, individuals with higher systolic blood pressure, poorer glucose regulation,

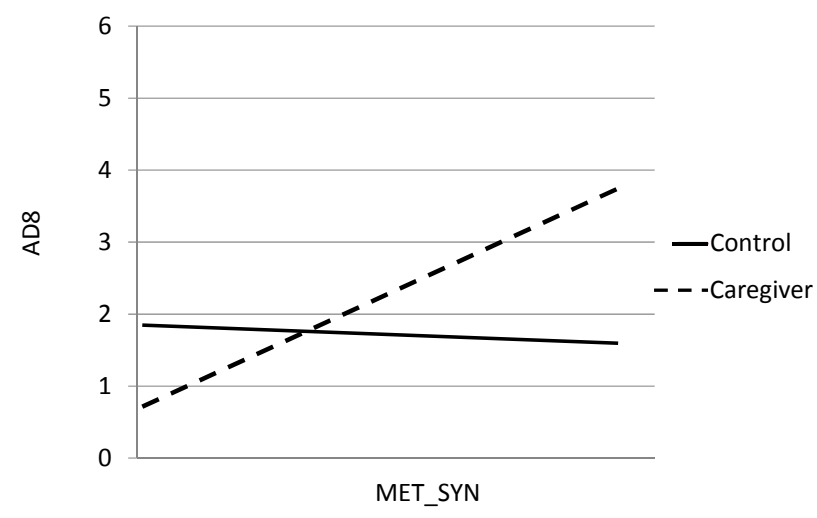

Figure 1. The relation between MET_SYN and AD8 ratings by group (caregivers and controls). 
central obesity, and increased lipid levels tended to have increased scores on a measure of cognitive decline. Therefore, as illustrated in the present study, models that include potential moderation should be used to provide a comprehensive picture of risk for disease.

The current findings support prior research that indicates metabolic regulation is related to cognitive decline e.g. $[7,8]$. Moreover, results from the present study indicate that the effect of poor metabolic regulation on cognitive decline is enhanced by the presence of a chronic stressor such as that of caregiving. A similar enhancement of the impact of CVD risk factors on CHD incidence has been shown for Type A behavior. Among men aged 50 - 59 in the Western Collaborative Group Study, the CHD rate in Type A men in the highest Framingham Study risk decile was 400/1000 compared to 270/1000 among Type B men in the highest risk decile [22].

The present results do not support a model that indicates metabolic factors mediate the relation between caregiving and decline in cognitive function, indeed, in the current data caregiving was not directly associated with decline. Our data conflicts with certain studies that have shown worsened cognition in caregivers, see [10]. One potential difference in our sample and a majority of the prior studies that have linked cognitive decline with the role of caregiving is that of the relation between the caregiver and the care recipient. A good deal of prior research has only included caregivers who are spouses of individuals with dementia, whereas, our study included both spouses and children of individuals with dementia. In the current study approximately half of the caregivers were children of the care recipient. This may be relevant as one of the mechanisms proposed to link decline with spousal caregiving is the potential for social isolation of the spousal caregiver-a factor that may be less prevalent in non-spouse caregivers. Furthermore, it has also been suggested that caregivers may have increased risk of cognitive decline than non-caregivers due to assortative mating [23], which would of course not be applicable to studies that include non-spouse caregivers.

There are plausible mechanisms that may account for the present findings. In the current study caregiving was not associated with cognitive decline as a main effect; however, in the presence of poor metabolic regulation caregiving was related to the increased ratings of decline. Thus, caregivers with a less healthy metabolic profile may have accelerated brain aging with consequent more rapid cognitive decline. Alternatively, metabolic traits vary according to forces-e.g. genetic, dietary-that do not necessarily involve caregiving. We have shown that caregivers with rs439401 TT alleles have metabolic trait levels reflecting poorer health [24,25]. Furthermore, rs439401 has been associated with Alzheimer's Disease
[26]. We can speculate that caregivers with TT and/or G genotypes had elevated metabolic traits, which then contributed to their cognitive decline.

There are limitations that should be noted when interpreting the present results. The participants in this study were recruited by various means and therefore may not be a representative sample. The present sample size was small, raising the possibility that our findings may not be stable across new samples. Additionally, given the crosssectional nature of the study, we relied on self-report of cognitive decline as opposed to detailed longitudinal neuropsychological observations. It is possible with a longitudinal observation over a period of years and with more detailed objective measures of cognitive function, cognitive deficits would be detected that may not surface in self appraisals of function.

As in any observational study, it is possible that other unmeasured factors may have influenced this relationship. Potential mechanisms have been proposed that may underlie associations between stressful circumstances and health outcomes. For example, poor health habits have been shown among caregivers [27]. In the present sample, the number of current smokers was small and assessment of other health habits, such as exercise and alcohol consumption, was limited, thus we were unable to adequately test additional mediational models concerning these health habits.

The fact that caregiving is associated with physical and mental suffering, as well as early mortality, has been well documented over the past two decades [28-31]. The present results indicate that caregiver stress may increase the association between metabolic risk factors and decline in cognitive functioning up to 8 years later.

The American Alzheimer's Association (http://www.alz.org/alzheimersdiseasefacts_and_figures.asp) reports that nearly 15 million individuals currently provide caregiving for patients with Alzheimer's disease or dementia, that $61 \%$ report high or very high rates of emotional stress of caregiving, and that $43 \%$ report high to very high rates of physical stress of caregiving. Thus, the impact of caregiving via increased levels of MET_SYN and cognitive decline are of considerable public health significance. Training caregivers in stress coping skills has the potential to reduce levels of MET_SYN components like blood pressure [32]. Such interventions aimed at reducing metabolic risk factors in caregiver populations may not only provide important dividends by reducing their likelihood of cardiovascular diseases, but it may also reduce risk for later dementias [33].

\section{Acknowledgements}

This research was supported by a pilot grant from the Bryan Alzheimer's Disease Research Center

P30-AG02837750; a grant from the National Institutes of 
Health PO1 HL036587-20; the Clinical Research Unit grant M01RR30; and a grant from the Alzheimer's Association IRG 08-89565.

\section{REFERENCES}

[1] L. Caswell, P. P. Vitaliano, K. Croyle, J. M. Scanlan, J. Zhang and A. Daruwala, "Negative Associations of Chronic Stress and Cognitive Functioning in Older Adult Spouse Caregivers," Experimental Aging Research, Vol. 29, No. 3, 2003, pp. 303-318. doi:10.1080/03610730303721

[2] S. Lee, I. Kawachi and F. Grodstein, "Does Caregiving Stress Affect Cognitive Function in Older Women?” The Journal of Nervous and Mental Disease, Vol. 192, No. 1, 2004, pp. 51-57. doi:10.1097/01.nmd.0000106000.02232.30

[3] M. E. de Vugt, J. Jolles, L. van Osch, et al., "Cognitive Functioning in Spousal Caregivers of Dementia Patients: Findings from the Prospective MAASBED Study," Age and Ageing, Vol. 35, No. 2, 2006, pp. 160-166. doi:10.1093/ageing/afj044

[4] M. C. Norton, et al., "Greater Risk of Dementia when Spouse Has Dementia? The Cache County Study,” Journal of the American Geriatrics Society, Vol. 58, No. 5, 2010, pp. 895-900. doi:10.1111/j.1532-5415.2010.02806.x

[5] P. P. Vitaliano, J. M. Scanlan, J. Zhang, M. V. Savage, I. B. Hirsch and I. C. Siegler, "A Path Model of Chronic Stress, the Metabolic Syndrome, and Coronary Heart Disease,” Psychosomatic Medicine, Vol. 64, No. 3, 2002, pp. 418-435.

[6] P. P. Vitaliano, J. M. Scanlan, I. C. Siegler, W. C. McCormick and R. H. Knopp, "Coronary Heart Disease Moderates the Relationship of Chronic Stress with the Metabolic Syndrome," Health Psychology, Vol. 17, No. 6, 1998, pp. 520-529,. doi:10.1037/0278-6133.17.6.520

[7] K. Yaffe, "Metabolic Syndrome and Cognitive Disorders: Is the Sum Greater Than Its Parts?” Alzheimer Disease and Associated Disorders, Vol. 21, No. 2, 2007, pp. 167171. doi:10.1097/WAD.0b013e318065bfd6

[8] K. Yaffe, A. L. Weston, T. Blackwell, et al., "The Metabolic Syndrome and Development of Cognitive Impairment among Older Women,” Archives of Neurology, Vol. 66, No. 3, 2009, pp. 324-328. doi:10.1001/archneurol.2008.566

[9] V. Frisardi, et al., "Metabolic-Cognitive Syndrome: A Cross-Talk between Metabolic Syndrome and Alzheimer's Disease,” Ageing Research Reviews, Vol. 9, No. 4, 2010, pp. 399-417. doi:10.1016/j.arr.2010.04.007

[10] P. P. Vitaliano, M. Murphy, H. M. Young, D. Echeverria and S. Borson, "Does Caregiving for a Spouse with Demential Promote Cognitive Decline? A Hypothesis and Proposed Mechanisms," Journal of the American Geriatrics Society, Vol. 59, No. 5, 2011, pp. 900-908. doi:10.1111/j.1532-5415.2011.03368.x

[11] R. B. Williams, "Psychosocial and Biobehavioral Factors and Their Interplay in Coronary Heart Disease,” Annual
Review of Clinical Psychology, Vol. 4, No. 1, 2008, pp. 349-365. doi:10.1146/annurev.clinpsy.4.022007.141237

[12] B. H. Brummett, C. M. Kuhn, S. H. Boyle, M. A. Babyak, I. C. Siegler and R. B. Williams, "Cortisol Responses to Emotional Stress in Men: Association with a Functional Polymorphism in the 5HTR2C Gene," Biological Psychology, Vol. 89, No. 1, 2012, pp. 94-98. doi:10.1016/j.biopsycho.2011.09.013

[13] B. H. Brummett, et al., "Neighborhood Characteristics Moderate Effects of Caregiving on Glucose Functioning," Psychosomatic Medicine, Vol. 67, No. 5, 2005, pp. 752758. doi:10.1097/01.psy.0000174171.24930.11

[14] J. E. Galvin, C. M. Roe, M. A. Coats and J. C. Morris, "Patient's Rating of Cognitive Ability," Archives of Neurology, Vol. 64, No. 5, 2007, pp. 725-730. doi:10.1001/archneur.64.5.725

[15] D. M. Nathan, D. E. Singer, K. Hurxthal and J. D. Goodson, "The Clinical Information Value of the Glycosylated Hemoglobin Assay,” New England Journal of Medicine, Vol. 310, No. 6, 1984, pp. 341-346. doi:10.1056/NEJM198402093100602

[16] J. E. Aikens, J. L. Wallander, D. S. Bell and J. A. Cole, "Daily Stress Variability, Learned Resourcefulness, Regimen Adherence, and Metabolic Control in Type I Diabetes Mellitus: Evaluation of a Path Model," Journal of Consulting and Clinical Psychology, Vol. 60, No. 1, 1992, pp. 113-118. doi:10.1037/0022-006X.60.1.113

[17] L. S. Griffith, B. J. Field and F. J. Lustman, "Life Stress and Social Support in Diabetes: Association with Glycemic Control,” International Journal of Psychiatry in Medicine, Vol. 20, No. 4, 1990, pp. 365-372. doi:10.2190/APH4-YMBG-NVRL-VLWD

[18] L. A. Jaber, N. J. Lewis, R. L. Slaughter and A. V. Neale, "The Effect of Stress on Glycemic Control in Patients with Type II Diabetes during Glyburide and Glipizide Therapy," Journal of Clinical Pharmacology, Vol. 33, No. 3, 1993, pp. 239-245.

[19] K. Khaw, N. Wareham, R. Luben, S. Bingham, S. Oakes and A. Welch, "Glycated Haemoglobin, Diabetes, and Mortality in Men in Norfolk Cohort of European Prospective Investigation of Cancer and Nutrition (EPIC-Norfolk),” British Medical Journal, Vol. 322, No. 15, 2001, pp. 15-18. doi:10.1136/bmj.322.7277.15

[20] P. Falke, F. Lindgarde and L. Stavenow, "Differences in Blood Viscosity, Glycosylated Hemoglobin and Platelet Count between Male Patients with Carotid Transient Ischemic Attacks and Minor Strokes,” Clinical Hemorheology and Microcirculation, Vol. 11, No. 1-2, 1991, pp. 35-40.

[21] J. E. Galvin, et al., "A Brief Informant Interview to Detect Dementia,” Neurology, Vol. 65, No. 4, 2005, pp. 559-564. doi:10.1212/01.wnl.0000172958.95282.2a

[22] R. J. Brand, R. H. Rosenman, R. I. Sholtz and M. Friedman, "Multivariate Prediction of Coronary Heart Disease in the Western Collaborative Group Study Compared to the Findings of the Framingham Study," Circulation, Vol. 53, No. 2, 1976, pp. 348-355. doi:10.1161/01.CIR.53.2.348 
[23] D. M. Buss, "Toward a psychology of Person-Environment (PE) Correlation: The Role of Spouse Selection," Journal of Personality and Social Psychology, Vol. 47, No. 2, 1984, pp. 361-377.

doi:10.1037/0022-3514.47.2.361

[24] S. I. Kring, et al., "Impact of Psychological Stress on the Associations between Apolipoprotein E Variants and Metabolic Traits: Findings in an American Sample of Caregivers and Controls,” Psychosomatic Medicine, Vol. 72, No. 5, 2010, pp. 427-433. doi:10.1097/PSY.0b013e3181de30ad

[25] S. I. Iqbal Kring, et al., "Associations between APOE Variants and Metabolic Traits and the Impact of Psychological Stress,” PLoS One, Vol. 6, No. 1, 2011, Article ID: e15745. doi:10.1371/journal.pone.0015745

[26] D. Harold, et al., "Genome-Wide Association Study Identifies Variants at CLU and PICALM Associated with Alzheimer's Disease,” Nature Genetics, Vol. 41, No. 10, 2009, pp. 1088-1093. doi:10.1038/ng.440

[27] J. K. Kiecolt-Glaser, J. R. Dura, C. E. Speicher, J. Trask and R. Glaser, "Spousal Caregivers of Dementia Victims: Longitudinal Changes in Immunity and Health," Psychosomatic Medicine, Vol. 53, No. 4, 1991, pp. 345-362.

[28] R. Schulz, A. T. O’Brien, J. Bookwala and K. Fleissner, "Psychiatric and Physical Morbidity Effects of Dementia Caregiving: Prevalence, Correlates, and Causes,” The Ge- rontologist, Vol. 35, No. 6, 1995, pp. 771-791. doi:10.1093/geront/35.6.771

[29] R. Schulz, P. Visintainer and G. M. Williamson, "Psychiatric and Physical Morbidity Effects of Caregiving,” Journals of Gerontology: Series B: Psychological Sciences and Social Sciences, Vol. 45, No. 5, 1990, pp. 181-191.

[30] R. Schulz and G. M. Williamson, "A 2-Year Longitudinal Study of Depression among Alzheimer's Caregivers," Psychology and Aging, Vol. 6, No. 4, 1991, pp. 569-578. doi:10.1037/0882-7974.6.4.569

[31] R. Schulz and P. R. Sherwood, "Physical and Mental Health Effects of Family Caregiving,” American Journal of Nursing, Vol. 108, No. 9, 2008, pp. 23-27. doi:10.1097/01.NAJ.0000336406.45248.4c

[32] V. P. Williams, et al., "Video-Based Coping Skills to Reduce Health Risk and Improve Psychological and Physical Well Being in Alzheimer's Disease Family Caregivers," Psychosomatic Medicine, Vol. 72, No. 9, 2010, pp. 897-904. doi:10.1097/PSY.0b013e3181fc2d09

[33] I. C. Siegler, H. B. Bosworth, B. H. Brummett and M. F. Elias, "Adult Development and Aging in Health Psychology,” In: A. M. Nezu, C. M. Nezu and P. A. Geller, Eds., Comprehensive Handbook of Psychology, Health Psychology, Wiley, New York, 2011, pp. 487-510. 